\title{
REVIEW
}

\section{Health consequences of obesity}

\section{J J Reilly, E Methven, Z C McDowell, B Hacking, D Alexander, L Stewart, C J H Kelnar}

Arch Dis Child 2003;88:748-752

The recent epidemic of childhood obesity' has raised concern because of the possible clinical and public health consequences. ${ }^{2}{ }^{3}$ However, there remains a widespread perception among health professionals that childhood obesity is a largely cosmetic problem, with minor clinical effects. No systematic review has yet focused on the diverse array of possible consequences of childhood obesity, though older non-systematic reviews are available. ${ }^{45}$ In addition, no review to date has considered the vast body of evidence on the health impact of childhood obesity which has been published recently. The aim of the present review was therefore to provide a critically appraised, evidence based, summary of the consequences of childhood obesity in the short term (for the child) and longer term (in adulthood).

\begin{abstract}
METHODS
Literature searching

We searched for papers using Medline, Embase, Cinahl, Healthstar, the Cochrane Library, and the internet from January 1981 to the end of December 2001. ${ }^{67}$ Literature searching was carried out by a specialist librarian. The search was supplemented by manual searching of reference lists from each relevant paper identified, as well as contents pages of 29 relevant specialist journals from January 1997 to December 2001. Further details of the search strategies used are available from the corresponding author. Papers which were identified were assessed for relevance to the question being addressed by a multidisciplinary group.
\end{abstract}

\section{Evidence appraisal}

The multidisciplinary group, all trained in critical appraisal, used Scottish Intercollegiate Guidelines (SIGN) methodology to appraise the published evidence. This approach has been described elsewhere. ${ }^{6-8}$ In brief, each paper identified as relevant was appraised independently by two reviewers. They agreed on a methodological quality rating using the methodology ${ }^{7}$ and hierarchy of study types published elsewhere. ${ }^{8}$ Where agreement was not obtained papers were referred to a third reviewer or to a specialist in critical appraisal methodology. The hierarchy of study types was as follows: level l evidence, systematic reviews, meta-analyses, and randomised controlled trials (RCTs); level 2, non-randomised intervention studies, observational and cohort studies; level 3, surveys and quasi-experimental studies; level 4, expert opinion. Studies considered eligible
Children, Yorkhill,

Glasgow G3 8SJ, UK

iir2y@clinmed.gla.ac.uk were rated for methodological quality by at least two independent reviewers using published criteria $^{6-8}$ : all or most methodological criteria met, low risk of bias; some criteria not fulfilled or study inadequately described, low risk of bias; few or no criteria fulfilled, high risk of bias. Conclusions of this review are based solely on evidence with low risk of bias, and references are cited for such evidence. The rationale for this approach is that recommendations based on evidence should be explicitly linked to the strength of the underlying evidence, and, where possible, based on high quality evidence. ${ }^{78}$ The nature of the question being addressed meant that most of the evidence considered was level 2 and 3. Reference details for studies which were excluded, and those which were appraised but rated as being of poor methodological quality, have not been cited here but are available from the corresponding author on request.

\section{Inclusion and exclusion criteria}

Studies were excluded from the appraisal if they did not define obesity in their subjects. This was relatively common in older literature. A number of other, largely older, studies did not describe how obesity was defined, or relied on subjective assessment for the definition, or subject self selection. The best available simple obesity definitions are based on body mass index (BMI) centile, ${ }^{6}$ and the present evidence appraisal is based largely on studies where obesity was defined in this way. In some of the studies we appraised, the Rohrer Index (weight/height ${ }^{3}$ ) was used to define obesity. We excluded case studies and reviews of case studies. We considered evidence from studies of subjects aged $1-18$ years. The evidence fell logically into two major categories: co-morbidities of childhood obesity in childhood (psychological; cardiovascular risk factors; other medical consequences); and consequences for the adult who was obese as a child or adolescent (socioeconomic effects; persistence of obesity; cardiovascular risk factors; adult morbidity and premature mortality).

\section{RESULTS}

Short term consequences of paediatric obesity

Psychological consequences

Nine studies identified from the search were excluded from our critical appraisal on the grounds that they did not define obesity in their subjects, or did not use an objective definition. Nine studies (cohort or cross sectional) were appraised, and five of these represented high quality evidence (table 1). We can conclude that obese children are more likely to experience 
Table 1 Consequences of childhood obesity in childhood: references appraised

\begin{tabular}{|c|c|c|}
\hline First author, year & Reference & Evidence level \\
\hline \multicolumn{3}{|l|}{ Psychological } \\
\hline Epstein, 1994 & Int J Eat Disord 15:151-7 & $2++$ \\
\hline Israel, 1985 & J Pediatr Psychol 10:449-59 & $2+$ \\
\hline Strauss, 2000 & Pediatrics 105:e 15 & $2+$ \\
\hline Erickson, 2000 & Arch Pediatr Adolesc Med 154:931-5 & $2+$ \\
\hline Phillips, 1998 & Int J Obes 22:287-93 & $2+$ \\
\hline \multicolumn{3}{|l|}{ Asthma } \\
\hline Figeroa-Munoz, 2001 & Thorax 56:133-7 & $2+$ \\
\hline Chinn, 2001 & Thorax 56:845-50 & $2+$ \\
\hline Belamerich, 2000 & Pediatrics 106:1436-41 & $2+$ \\
\hline Castro-Rodriguez, 2001 & Am J Respir Crit Care Med 163:1344-9 & $2+$ \\
\hline Von Mutius, 2001 & Thorax 56:835-8 & $2+$ \\
\hline \multicolumn{3}{|l|}{ Chronic inflammation } \\
\hline Ford, 2001 & J Pediatr 138:486-92 & $2+$ \\
\hline Cook, 2000 & Atherosclerosis 149:139-50 & $2+$ \\
\hline Visser, 2001 & Pediatrics 107 & $2+$ \\
\hline \multicolumn{3}{|l|}{ Other } \\
\hline Hypponen, 2000 & Diabet Care 23:1755-60 & $2++$ \\
\hline Riddiford-Harland, 2000 & Int J Obes 24:541-4 & $2+$ \\
\hline Dietz, 1998 & Pediatrics 101:518-25 & 4 \\
\hline \multicolumn{3}{|c|}{ Cardiovascular risk factors } \\
\hline Martin, 1973 & J Pediatr 82:192-201 & $2++$ \\
\hline Smoak, 1987 & Am J Epidemiol 125:364-72 & $2+$ \\
\hline Freedman, 1987 & Am J Clin Nutr 46:403-10 & $2++$ \\
\hline Freedman, 1989 & Am J Clin Nutr 50:930-9 & $2++$ \\
\hline Wattigney, 1991 & Arch Intern Med 151:2017-22 & $2++$ \\
\hline Le Stunff, 1994 & Diabetes 43:696-702 & $2+$ \\
\hline Shea, 1994 & Pediatrics $\mathbf{8 4 : 4 6 5 - 7 0}$ & $2+$ \\
\hline Wabitsch, 1994 & Am J Clin Nutr 60:54-60 & $2++$ \\
\hline Daniels, 1995 & Circulation 92:3249-54 & $2++$ \\
\hline Monti, 1995 & Diabetologia 38:739-47 & $2++$ \\
\hline Urbina, 1995 & Circulation $91: 2400-6$ & $2++$ \\
\hline McGill, 1995 & Arterioscler Thromb Biol 15:431-40 & $2++$ \\
\hline Gidding, 1995 & J Pediatr 127:868-74 & $2++$ \\
\hline Caprio, 1996 & Am J Clin Nutr 64:12-17 & $2++$ \\
\hline Bao, 1997 & JAMA 278:1749-54 & $2++$ \\
\hline Nain-Feng, 1998 & Am J Clin Nutr 67:1 141-6 & $2++$ \\
\hline Gutin, 1998 & J Pediatr 132:1023-7 & $2++$ \\
\hline Berenson, 1998 & N Engl J Med 338:1050-6 & $2++$ \\
\hline Washino, 1999 & Paediatrics Int $41: 260-5$ & $2+$ \\
\hline Freedman, 1999a & Am J Clin Nutr 69:308-17 & $2+$ \\
\hline Freedman, $1999 \mathrm{~b}$ & Pediatrics 103:1 175-82 & $2++$ \\
\hline Morrison, 1999a & J Pediatr 135:451-7 & $2++$ \\
\hline Morrison, $1999 b$ & J Pediatr 135:458-64 & $2++$ \\
\hline Cowin, 2000 & Int J Obes 24:330-9 & $2+$ \\
\hline Higgins, 2001 & Obes Res 9:233-40 & $3+$ \\
\hline Maffeis, 2001 & Obes Res 9:179-87 & $2++$ \\
\hline Young, 2000 & J Pediatr 136:365-9 & $3+$ \\
\hline Tounian, 2001 & Lancet 358: 1400-4 & $3+$ \\
\hline Qing He, 2000 & Hypertension 36: 165-70 & $2+$ \\
\hline Mamalakis, 2001 & Prev Med 33:525-535 & $2+$ \\
\hline Chu, 2000 & Int J Obes 24: 1265-71 & $2+$ \\
\hline
\end{tabular}

psychological or psychiatric problems than non-obese children, that girls are at greater risk than boys, and that risk of psychological morbidity increases with age. Low self esteem and behavioural problems were particularly commonly associated with obesity. For example, Strauss 9 found that $34 \%$ of obese (defined as BMI $>95$ th centile), white, 13-14 year old girls had low self esteem (defined as $<10$ th centile) compared to $8 \%$ of non-obese white girls. However, there was also some evidence from our review that parental psychological or psychiatric problems might have a greater influence on the child's psychological morbidity than obesity or gender.

\section{Cardiovascular risk factors in childhood}

A total of 38 eligible studies were identified which reported on the association between objectively defined indices of obesity (notably BMI), and/or central obesity (for example, waist circumference) and cardiovascular risk factors measured in childhood. Four of the studies identified were excluded (case studies, and/or studies with no obesity definition). The remaining 34 were critically appraised, and only three of these were rated as low quality evidence. The evidence, cited in table 1 , therefore consists of a large number of high quality studies which consistently showed associations between obesity (often defined as BMI $>85$ th or 95th centiles) and most of the major cardiovascular risk factors: high blood pressure; dyslipidaemia; abnormalities in left ventricular mass and/or function; abnormalities in endothelial function; and hyperinsulinaemia and/or insulin resistance. For example, Freedman and colleagues, ${ }^{10}$ using an obesity definition of BMI $>95$ th centile in a sample of 5-10 year olds from the USA, reported 
Table 2 Long term consequences of childhood obesity: references appraised

\begin{tabular}{|c|c|c|}
\hline First author, year & Reference & Evidence level \\
\hline \multicolumn{3}{|c|}{ Socioeconomic effects } \\
\hline Sargent, 1994 & Arch Pediatr Adolesc Med 148:681-7 & $2+$ \\
\hline Gortmaker, 1993 & N Engl J Med 329: 1008-12 & $2+$ \\
\hline \multicolumn{3}{|c|}{ Obesity persistence } \\
\hline Serdula, 1993 & Prev Med 22:167-77 & $2+$ \\
\hline Lake, 1997 & Am J Clin Nutr 77:376-81 & $2+$ \\
\hline Whitaker, 1997 & N Engl J Med 377:869-73 & $2+$ \\
\hline Laitinen, 2001 & Am J Clin Nutr 74:287-94 & $2++$ \\
\hline Hardy, 2000 & Int J Obes 24:725-34 & $2+$ \\
\hline Mo Suwan, 2000 & Int J Obes 24:1642-7 & $2+$ \\
\hline Freedman, 2001 & Pediatrics 108:712-18 & $2++$ \\
\hline \multicolumn{3}{|c|}{ Adult morbidity/mortality } \\
\hline Hoffmans, 1988 & J Clin Epidemiol 4:749-56 & $2+$ \\
\hline \multicolumn{3}{|c|}{ Cardiovascular risk factors } \\
\hline Smoak, 1987 & Am J Epidemiol 125:364-72 & $2+$ \\
\hline Wattigney, 1991 & Arch Intern Med 151:2017-22 & $2++$ \\
\hline McGill, 1995 & Arterioscler Thromb Biol 15:431-40 & $2++$ \\
\hline Bao, 1994 & Arch Intern Med 154:1842-7 & $2++$ \\
\hline Bao, 1997 & JAMA 278:1749-54 & $2++$ \\
\hline Berenson, 1998 & Am J Epidemiol 125:364-72 & $2++$ \\
\hline Wattigney, 1995 & Prev Med 24:617-26 & $2++$ \\
\hline Freedman, 2001 & Pediatrics 108:712-18 & $2++$ \\
\hline
\end{tabular}

significant odds ratios for raised diastolic blood pressure (OR 2.4 , raised blood pressure defined as $>95$ th centile), raised systolic blood pressure (OR 4.5, raised defined as >95th centile), raised LDL cholesterol (OR 3.0, raised defined as $>130 \mathrm{mg} / \mathrm{dl}$ ), low HDL cholesterol (OR 3.4, low defined as $<35$ $\mathrm{mg} / \mathrm{dl}$ ), raised triglycerides (OR 7.1, raised defined as $>130$ $\mathrm{mg} / \mathrm{dl}$ ), and high fasting insulin concentration (OR 12.1, high defined as $>95$ th centile). In the same study, Freedman and colleagues $^{10}$ also found that $58 \%$ of obese $5-10$ year olds had at least one of these five cardiovascular risk factors, $25 \%$ had two or more, and the odds ratios for having two and three of the five risk factors (relative to non-obese 5-10 year old children in the cohort) were 9.7 and 43.5 respectively. Many studies have observed significant "clustering" of cardiovascular risk factors (the aggregation of risk factors in the same individual) with paediatric obesity, and it has become clear that the extent of asymptomatic atherosclerotic lesions in childhood and adolescence is predicted by the number of cardiovascular risk factors present. ${ }^{11}$ In summary, it is now well established that childhood obesity (and central adiposity) have adverse effects on the cardiovascular system which are similar to those well known in adults. Expert opinion has also reached the same conclusion consistently, and has pointed out the similarities between children and adults in the lifestyle factors and biological mechanisms by which obesity promotes cardiovascular risk. ${ }^{4}{ }^{12-15}$

\section{Other clinical consequences in childhood}

A total of 13 of the studies which were identified as potentially relevant were excluded on a variety of grounds: they did not use an acceptable definition of obesity; did not include a control group; or used very small sample sizes. Expert opinion (evidence level 4) has also concluded that paediatric obesity has a variety of adverse health consequences. ${ }^{45}$

Of the remaining evidence, six papers were identified which reported associations between paediatric obesity and asthma (risk of asthma or severity of pre-existing asthma). Five of these six (table 1) were rated as high quality evidence and they reported fairly consistently that asthma was a comorbidity of childhood obesity. This evidence included one longitudinal study which reported that becoming obese significantly increased risk of developing asthma symptoms in girls who were apparently non-asthmatic at baseline. ${ }^{15}$

A further seven studies which reported on a variety of other possible clinical consequences of childhood obesity met our entry criteria and were appraised. Two studies were rated as being of low methodological quality and so not included in this review. Five of the studies represented high quality evidence. Hypponen and colleagues ${ }^{16}$ found that paediatric obesity was associated with a more than twofold risk of developing type 1 diabetes (table 1). Three high quality studies have suggested that paediatric obesity may be associated with low grade systemic inflammation. For example, Visser and colleagues $^{17}$ found, in 3512 children aged 8-16 years old participating in the third US National Health and Nutrition Examination Survey, that overweight (defined as BMI $>85$ th centile) was significantly and independently associated with increased serum $\mathrm{C}$ reactive protein concentration. The adjusted odds ratios, reported relative to children with BMI $<85$ th centile, were 3.74 in boys and 3.17 in girls. One high quality study found significant associations between obesity and abnormalities of foot structure (table 1).

\section{Long term consequences of paediatric obesity \\ Social and economic effects}

Three studies were identified; two met our entry criteria and so were critically appraised (table 2). Both longitudinal studies presented high quality evidence that obesity in adolescence/ young adulthood has adverse effects on social and economic outcomes in young adulthood (for example, income, educational attainment), controlled for a number of other variables, including intelligence quotient. There was some evidence that these effects might be more marked in women than men. For example, British girls born in 1958 who had BMI $>90$ th centile when studied at age 16 had significantly lower income than girls with BMI <90th centile (by $7 \%$ on average) at age 23 , controlled for social class and intelligence quotient. ${ }^{18}$

\section{Persistence of obesity from childhood}

We identified 11 eligible studies, four of which were rated as being of low methodological quality and so are not included in 
this review. The remaining seven studies (table 2) were of high quality and showed a tendency for childhood obesity to persist. In six of the seven studies persistence was reported into adulthood, though one study focused on persistence of obesity from early childhood into adolescence. Persistence of childhood obesity into adulthood was substantially more likely where children had at least one obese parent, where obesity was more severe (for example, defined as BMI >95th centile compared to BMI $>85$ th centile), and present at older ages. Adolescent obesity is probably even more likely to persist into adulthood than childhood obesity. ${ }^{19}$ Whitaker and colleagues $^{19}$ found that $69 \%$ of obese (defined as BMI $>95$ th centile) 6-9 year olds in the USA were obese as adults (OR 18.5 , using 6-9 year olds with BMI $<85$ th centile as the reference group). In the same cohort, $83 \%$ of obese (BMI $>95$ th centile) 10-14 year olds became obese adults (OR 44.3, reference group BMI $<85$ th centile).

Most of the available evidence on the persistence of childhood obesity was based on older studies, when prevalence of parental obesity was much lower. It is worth noting that persistence of childhood obesity into adulthood should now be more likely than in the past, given the much higher prevalence of adult obesity in contemporary populations. Estimates of obesity persistence based on cohorts of children born many years ago vary as a result of a number of factors, but typically suggest that around $40-70 \%$ of obese pre-pubertal children will become obese adults. These studies will underestimate the magnitude of the effect for modern children in more "obesogenic" environments. For example, Freedman and colleagues $^{20}$ found that $77 \%$ of obese (BMI >95th centile) children were obese (BMI $>30)$ as adults.

\section{Impact of childhood obesity on adult morbidity and risk of premature mortality}

Four relevant studies were identified. One was excluded and two others were considered to have a high risk of bias. One remaining cohort study, ${ }^{21}$ of high methodological quality (table 2), found that a BMI of $>25 \mathrm{~kg} / \mathrm{m}^{2}$ at age 18 was associated with significantly increased mortality within 20 years of follow up. By the 32 year follow up, a BMI of greater than or equal to $26 \mathrm{~kg} / \mathrm{m}^{2}$ at age 18 years was associated with significantly increased mortality risk (RR 1.95 , with BMI $19 \mathrm{~kg} / \mathrm{m}^{2}$ as the reference group). Collecting evidence which addresses long term effects of childhood or adolescent obesity is clearly difficult, but there is a need for more research in this area. It is worth noting that all of the evidence appraised on long term morbidity/mortality risk suggested adverse effects of childhood or adolescent obesity. Evidence on cardiovascular risk factors (see below) predicts increased adult cardiovascular morbidity and mortality arising from childhood obesity; this conclusion was also reached by all expert opinion..$^{3-5} 1214$

\section{Cardiovascular risk factors in adulthood}

Nine studies tested for relations between paediatric obesity and adult cardiovascular risk factors. One study was of poor methodological quality, leaving eight high quality studies (table 2). Most of these were reports from the Bogalusa Heart Study. Most of the publications reported significant associations between atherogenic profiles associated with obesity in childhood and those in adulthood. We can conclude that obesity mediated cardiovascular morbidity in adulthood can have its origins in childhood obesity, and that the magnitude of this problem is likely to be much greater now than in the past, following the epidemic of childhood obesity. Expert opinion (evidence level 4) has also reached similar conclusions consistently. - $^{12} 14$ However, distinguishing differences in adult cardiovascular risk between adults who were obese as children, and those who were non-obese as children is not straightforward..$^{20}$

\section{DISCUSSION}

The present review was the first to systematically search and critically appraise the substantial literature on the health impact of paediatric obesity. Addressing this issue from a critical, evidence based, perspective is particularly important now that obesity is so common in children and adolescents worldwide, and the demands for clinical and public health action are increasing. ${ }^{23}$ Any such actions should be informed by appraisal of the evidence base, and the health impact of paediatric obesity is of fundamental importance. Clearly identifying the evidence base is also important to address the widespread perception among health professionals that obesity in childhood is relatively unimportant (a largely cosmetic problem), or that it matters only in the context of the risk it provides for later (adult) health. The present review has shown that paediatric obesity is likely to be a major cause of ill health in adulthood, but also that it contributes substantially to illness in childhood. Finally, systematic review and critical appraisal is very effective at identifying research needs, ${ }^{6}$ and the present review has identified a number of weaknesses in the evidence on the health impact of childhood obesity.

Some co-morbidities of obesity in childhood, such as type II diabetes, ${ }^{22}$ polycystic ovarian syndrome, and various other conditions (a variety of respiratory, orthopaedic, and hepatic abnormalities) are now fairly well established, although we found that the evidence base for these was somewhat limited. This reflected a combination of factors, notably the rarity of some of these disorders in the past, a substantial body of fairly consistent but poorer quality evidence for others, and/or a substantial quantity of evidence from study designs which were ineligible in the present review (case studies). It was obvious from our review that both the quantity and quality of published evidence on the health impact of paediatric obesity is increasing, and our understanding of this subject is likely to improve rapidly in the next few years. It is also worth noting that conclusions from studies rated as being of poorer methodological quality were generally very similar to conclusions reached by studies which were more rigorously conducted or reported.

The design of the studies considered by the present review meant that in most cases we have summarised evidence which identified associations between obesity and morbidity. These studies cannot provide definitive evidence of causation or the direction of causation. However, for the studies presented in the present review, study design and analysis has been rigorous, a large body of consistent evidence exists in most cases, and there are plausible causal mechanisms which can explain why obesity might be associated with the morbidity. Where these conditions apply we can be more confident that obesity causes morbidity. We have been unable to present data on the magnitude of many of the co-morbidities, and clearly generalisable estimates of the strength of many of the associations reported. This reflects both a lack of data on the prevalence of many of the co-morbidities in the paediatric population, and the heterogeneity of the studies identified in the review process. For example, studies have used a wide variety of obesity definitions and cut off points, and a number of different definitions of the co-morbidities studied. Where possible we have given examples of the "effect size", based on the highest quality studies we identified.

Most of the studies reviewed here used BMI percentile cut offs to define obesity. Most used population reference data, though some used internally generated BMI percentile cut off points. The present review has therefore provided further evidence of the "external validity" of such approaches to defining childhood obesity. The present review has provided a mass of evidence that the commonly used cut offs of BMI $>85$ th and $>95$ th centiles are biologically/clinically meaningful, and are not arbitrary. Furthermore, our earlier systematic review found that obesity definitions such as BMI $>95$ th centile had 
low to moderate sensitivity and high specificity, and can be used clinically to identify the fattest children in the population with confidence. ${ }^{63}$ The optimal definition of childhood obesity is still being debated, ${ }^{24}{ }^{25}$ but it is now evident that there are strong relations between BMI centile cut offs in childhood and short and long term morbidity.

\section{Conclusions}

Childhood obesity has significant adverse effects on health in childhood. Psychological morbidity is likely to be the most widespread health impact in childhood. ${ }^{4}$ A large body of high quality evidence has shown that childhood obesity is strongly associated with the presence and clustering of cardiovascular risk factors in childhood.

A number of long term adverse effects of childhood obesity are now well established. The socioeconomic impact of obesity in adolescence/young adulthood is considerable, but little known. Obesity in childhood tends to persist into adulthood. Cardiovascular effects of obesity in childhood persist and this predicts a strong link between childhood obesity and morbidity/mortality in adulthood, which should be reflected in increased cardiovascular morbidity in future, as the current generation of obese children become adults. Taken together, this evidence makes a strong case for greater efforts directed at the prevention and treatment of childhood obesity.

\section{ACKNOWLEDGEMENTS}

We thank Francesca Chappell for her efforts in literature searching. Work for this review contributed to the Scottish Intercollegiate Guidelines Network (SIGN), evidence based guideline on "Obesity in Children and Young People". ${ }^{26}$

\section{Authors' affiliations}

J J Reilly, Z C McDowell, University of Glasgow Division of Developmental Medicine, Royal Hospital for Sick Children, Yorkhill, Glasgow G3 8SJ

E Methven, The Cander Centre, 17 King Street, Stonehouse, Lanarkshire ML9 3EQ

B Hacking, Department of Oncology, Western General Hospital, Edinburgh

D Alexander, Nethertown Surgery, Elliot Street, Dunfermline KY 11 4TF L Stewart, Department of Dietetics, Royal Hospital for Sick Children, 20 Sylvan Place, Edinburgh EH9 IUW

C J H Kelnar, Section of Child Life \& Health, Department of Reproductive and Developmental Sciences, University of Edinburgh, Royal Hospital for Sick Children, 20 Sylvan Place, Edinburgh EH9 IUW

\section{REFERENCES}

1 Reilly JJ, Dorosty AR. Epidemic of obesity in UK children. Lancet 1999:354:1874-5.

2 Koplan JP, Dietz WH. Caloric imbalance and public health policy. JAMA 1999;282:1579-81.
3 Sokol RJ. The chronic disease of childhood obesity: the sleeping giant has awakened. J Pediatr 2000;136:711-13.

4 Dietz WH. Health consequences of obesity in youth: childhood predictors of adult disease. Pediatrics 1998;101:518-25.

5 Slyper AH. Childhood obesity, adipose tissue distribution, and the pediatric practioner. Pediatrics 1998;102:e4

6 Reilly JJ, Wilson M, Summerbell CD, et al. Obesity diagnosis, prevention, and treatment: evidence based answers to common questions. Arch Dis Child 2002;86:392-5

7 SIGN. SIGN 50-A guideline developers handbook. Scottish Intercollegiate Guidelines Network, 2002. www.sign.ac.uk/guidelines/ fulltext/50/index.html.

8 Harbour R, Miller J. A new system for grading recommendations in evidence based guidelines. BM 2001;323:334-6

9 Strauss RS. Childhood obesity and self-esteem. Pediatrics 2000;105:e15.

10 Freedman DS, Dietz WH, Srinivasan SR, et al. The relation of overweight to cardiovascular risk factors among children and adolescents: the Bogalusa Heart Study. Pediatrics 1999;103:1 175-82.

11 Berenson GS, Srinivasan SR, Bao W, et al. Association between multiple cardiovascular risk factors and atherosclerosis in children and young adults. N Engl I Med 1998:338:1650-6.

12 Williams CL, Hayman LL, Daniels SR, et al. Cardiovascular health in childhood: a statement for health professionals from the committee on atherosclerosis, hypertension, and obesity in the young. Circulation 2002; 106: 143-60.

13 Barlow SE, Dietz WH. Obesity evaluation and treatment: expert committee recommendations. Pediatrics 1998;102:e3

14 Giddings S, Leibel RL, Daniels SR, et al. Understanding obesity in youth American Heart Association Scientific Statement. Circulation 1996;94:3383-7.

15 Castro-Rodriguez JA, Holberg CJ, Morgan WJ, et al. Increased incidence of asthma like symptoms in girls who become overweight or obese during the school years. Am J Respir Crit Care Med 2001;163:134-9.

16 Hypponen E, Virtanen S, Kenward MG, et al. Obesity, increased linear growth, and risk of type I diabetes in children. Diabetes Care 2000:23:1755-60.

17 Visser M, Bouter LM, McQuillan GM, et al. Low-grade systemic inflammation in overweight children. Pediatrics 2001;107:e13.

18 Sargent JD, Blanchflower DG. Obesity and stature in adolescence and earnings in young adulthood. Arch Pediatr Adolesc Med 1994:148:681-7.

19 Whitaker RC, Wright JA, Pepe MS, et al. Predicting obesity in young adulthood from childhood and parental obesity. N Engl J Med 1997;337:869-73.

20 Freedman DS, Kettel-Khan L, Dietz WH, et al. Relationship of childhood obesity to coronary heart disease risk factors in adulthood: the Bogalusa Heart Study. Pediatrics 2001;108:712-18.

21 Hofmanns MD, Kromhout D, de Lezanne-Coulander C. The impact of body mass index of 78,612 18 year old Dutch men on 32 year mortality from all causes. J Clin Epidemiol 1988;41:749-56.

22 Pinhias-Hamiel O, Dolan LM, Daniels SR, et al. Increased incidence of non-insulin dependent diabetes mellitus among adolescents. J Pediatr 1996; 128:608-15

23 Reilly JJ, Dorosty AR, Emmett PM, ALSPAC Study Team. Identification of the obese child: adequacy of the body mass index for clinical practice and epidemiology. Int J Obes Relat Metab Disord 2000:24:1623-7.

24 Reilly JJ. Assessment of childhood obesity: national or international reference data? Obes Res 2002;10:838-40.

25 Jebb SA, Prentice AM. Single definition of overweight and obesity should be used. BM 2001;323:999.

26 Scottish Intercollegiate Guidelines Network. Obesity in children and young people: an evidence based clinical guideline, 2003. www.sign.ac.uk. 